\title{
Particle acceleration by sound waves generated in the shock downstream region
}

\author{
Shota L. Yokoyama ${ }^{a, *}$ and Yutaka Ohira ${ }^{a}$ \\ ${ }^{a}$ Department of Earth and Planetary Science, The University of Tokyo, \\ 7-3-1 Hongo, Bunkyo-ku, Tokyo, 113-0033 Japan \\ E-mail: s_yokoyama@eps.s.u-tokyo.ac.jp, y.ohira@eps.s.u-tokyo.ac.jp
}

Our previous work revealed that the energy spectrum of cosmic rays produced by the standard diffusive shock acceleration (DSA) can be modified by downstream sound waves originated from inhomogeneity of the shock upstream medium. However, the background plasma was described by linear analytical solutions and nonlinear evolution of sound waves was not included. In this work, we investigate how nonlinear effects such as steepening and dissipation of sound waves affect particle acceleration by using test-particle simulations. First, we imitate weak shock waves steepened from sound waves by analytical sawtooth waves and show that large-scale waves can efficiently accelerate particles earlier than they dissipate. Next, we numerically solve shock propagation into an inhomogeneous medium and discuss particle acceleration by downstream waves. Our results imply additional acceleration by downstream sound waves works even when they evolve nonlinearly.

$37^{\text {th }}$ International Cosmic Ray Conference (ICRC 2021)

July 12 th - 23rd, 2021

Online - Berlin, Germany

\footnotetext{
${ }^{*}$ Presenter
} 


\section{Introduction}

Diffusive shock acceleration (DSA) in supernova remnants (SNRs) is believed to be one of the most plausible mechanisms by which energize galactic cosmic rays (CRs) [e.g. 2]. However, some problems are pointed out for this picture and it needs some modification $[5,6]$. For example, the maximum energy achievable by the standard DSA in typical supernova remnants does not reach the highest energy of galactic CRs (that is, knee energy). DSA also cannot explain the spectral index of CRs observed on the Earth and the diversity of spectral indices of radio synchrotron emissions from SNRs, since the standard DSA uniquely predicts a power-law momentum distribution $\mathrm{d} n / \mathrm{d} p \propto p^{-s}$ with spectral index $s=2$ for strong shocks.

Although in the conventional theory of DSA, the upstream medium was assumed to be uniform, it is natural to consider inhomogeneous medium and indeed the existence of various scales of density fluctuations is confirmed by observations [e.g. 1,4]. We have investigated the influence of medium inhomogeneity on particle acceleration using test-particle simulations and revealed that sound waves generated from the interaction between shock waves and upstream density fluctuations can cause additional particle acceleration in the shock downstream region $[13,14]$. However, in those works, fluctuations in the background plasma are treated as linear perturbations. It is still not unclear how medium inhomogeneity affects particle acceleration in more realistic situations.

In this study, we examine the effect of the nonlinear evolution of sound waves and its impact on particle acceleration by solving both test-particle motion and fluid equations numerically. Before that, we imitate the weak shock waves produce by steepening of sound waves by analytical sawtooth waves and investigate particle acceleration in such waves. In Section 2, we review our previous works and clarify the problems. We briefly introduce the numerical methods used in our simulations in Section 3. The results of simulations are shown in Section 4 and discussed in Section 5.

\section{Review}

In [14], we treated the background plasma which determines the shock structure as a fluid and the upstream fluctuation as a linear monochromatic entropy mode. That is, we assumed the following conditions for the shock upstream: $\rho(x, t)=\rho_{1}+\delta \rho_{1} \sin \left[k_{1}\left(x-u_{1} t\right)\right], u(x, t)=u_{1}$, and $P(x, t)=P_{1}$, where $\rho, u$, and $P$ mean the density, shock normal component of velocity measured in the shock rest frame, and pressure of the background plasma, respectively. $\delta \rho_{1}$ and $k_{1}$ are the amplitude and wavenumber of the upstream entropy mode. Subscript 1 is used for the upstream quantities, while 2 is used for downstream ones. Thanks to this treatment, we were able to relate the downstream quantities to the upstream ones analytically, following the linear analysis of [7]. We showed that sound waves originated from upstream medium inhomogeneity can accelerate particles in the shock downstream region. Acceleration mechanism by downstream sound waves is identified as second-order acceleration by large-scale compressible turbulence, which is discussed in e.g. [9]. Particles are stochastically accelerated by local velocity differences produced by downstream sound waves. The acceleration time-scale $t_{\text {acc,2nd }}$ in which this mechanism works is estimated in [14].

However, due to the linear approximation, sound waves generated in the shock downstream region extended infinitely far without any damping. Considering the nonlinear behavior of sound waves, they steepen into weak shock waves and dissipate in some finite time scale. This time-scale 
$t_{\text {dis }}$ can be evaluated as follows [10].

$$
\begin{aligned}
t_{\mathrm{dis}}=\frac{\lambda_{\delta u_{2}}}{\delta u_{2}} & =\frac{r\left(1+2 M_{2}+M_{1}^{-2}\right)}{(r-1) M_{2}}\left(\frac{u_{\mathrm{sh}}}{c}\right)^{-1}\left(\frac{\lambda_{\delta u_{2}}}{\lambda_{\mathrm{mfp}}\left(p_{0}\right)}\right)\left(\frac{\delta \rho_{1}}{\rho_{1}}\right)^{-1} \tau_{\mathrm{sc}}\left(p_{0}\right) \\
& \simeq 5.6\left(\frac{u_{\mathrm{sh}}}{c}\right)^{-1}\left(\frac{\lambda_{\delta u_{2}}}{\lambda_{\mathrm{mfp}}\left(p_{0}\right)}\right)\left(\frac{\delta \rho_{1}}{\rho_{1}}\right)^{-1} \tau_{\mathrm{sc}}\left(p_{0}\right) .
\end{aligned}
$$

$r$ and $M$ are the compression ratio $\rho_{2} / \rho_{1}$ and sound Mach number, respectively. In the second equal sign, we used the linear estimation by [7] for the amplitude of velocity fluctuations by downstream sound waves $\delta u_{2}$. And in the last similarity, numerical values for strong shock limit $r \rightarrow 4$ are used. The wavelength of sound waves $\lambda_{\delta u_{2}}$ and the dissipation time-scale $t_{\text {dis }}$ are normalized by the scattering mean free path $\lambda_{\mathrm{mfp}}(p)$ and scattering time $\tau_{\mathrm{sc}}(p)$ of particles with injection momentum $p_{0}$. In [14], this time-scale is compared to $t_{\mathrm{acc}, 2 \mathrm{nd}}$ and it is concluded that sound waves dissipate earlier than the second-order process works. On the other hand, acceleration by weak shock waves generated by steepening is not discussed quantitatively there. Therefore, whether the nonlinear evolution of sound waves impedes or promotes downstream acceleration is still unknown even for the one-dimensional monochromatic case. In this work, we tackle this problem by test-particle simulations.

\section{Test-particle simulations}

To model the particle motion in a turbulent magnetic field, we treat particles as test particles and their motion as isotropic diffusion in the local rest frame of background plasma. Particles are continuously injected at the main shock with initial momentum $p_{0}$. In each time step, we follow the following operations; (i) translation of particle positions, (ii) obtaining the plasma velocity at the new particle positions, (iii) Lorentz transformation to the local plasma rest frame, (iv) isotropic diffusion, (v) inverse Lorentz transformation to the original frame.

When we investigate particle acceleration under sawtooth waves generated from steepening of sound waves, we use the following analytical fluid fields.

$$
u(x, t)= \begin{cases}\delta u_{2} \sin \left[k_{2}\left(x-v_{\mathrm{s} 2} t\right)\right] & \text { (sinusoidal wave) } \\ \sqrt{6} \delta u_{2}\left[\left(x-v_{\mathrm{s} 2} t\right) / \lambda_{\delta u_{2}}-\text { floor }\left(\left(x-v_{\mathrm{s} 2} t\right) / \lambda_{\delta u_{2}}+0.5\right)\right] & (\text { sawtooth wave })\end{cases}
$$

where floor $(x)$ is the floor function which returns the greatest integer less than $x . k_{2}=2 \pi / \lambda_{\delta u_{2}}$ and $v_{\mathrm{s} 2}$ are the wavenumber and sound velocity of the downstream sound waves, respectively. The numerical factors appeared in the above equation are introduced to guarantee that energy density of the sawtooth waves is equal to that of the sinusoidal waves. In Figure 1, these analytical fields are plotted.

For simulations that numerically solve both particle motion and fluid equations, we adopt a Godunov-type scheme. Riemann problems are approximately solved by the HLLC method [e.g. 12] and fifth-order accuracy in space is achieved by the MP5 scheme [11]. Time width $\Delta t$ of each time step is calculated by the minimum of that determined by the CFL condition and particle scattering time. The velocity field calculated in this manner is referred to in step (ii) of the particle part. 


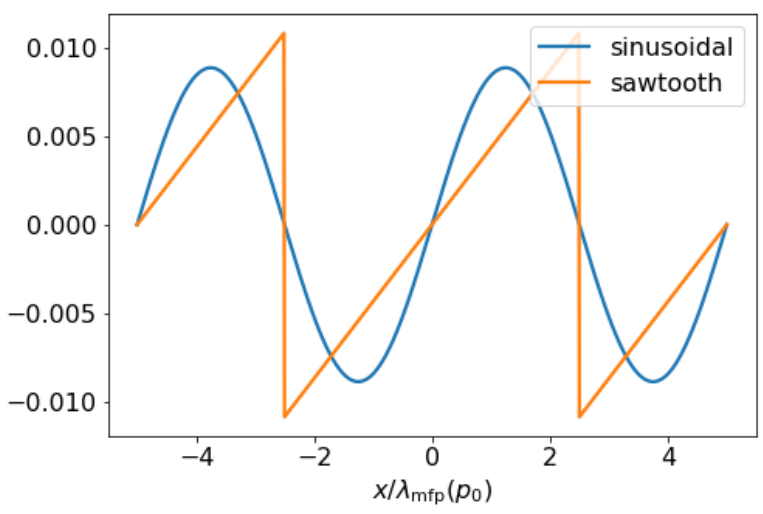

Figure 1: Analytical velocity fields.

\section{Results}

\subsection{Particle acceleration by sawtooth shock waves}

In order to evaluate particle acceleration under weak shock-train produced from steepening of sound waves, we use the analytical fluid field introduced in Equation (3) and shown in Figure 1. We continuously inject particles at $x=0$ and vary the wavelength of waves to $\lambda_{\delta u_{2}}=10,10^{2}, 10^{3}$, and, $10^{4} \lambda_{\mathrm{mfp}}\left(p_{0}\right)$. The amplitude of sound waves $\delta u_{2}$ and sound velocity $v_{\mathrm{s} 2}$ are calculated so as to coincide with downstream quantities when $u_{\mathrm{sh}}=0.1 \mathrm{c}$ and $\delta \rho_{1}=0.5 \rho_{1}$.

The resulted momentum spectra are shown in Figure 3. For short wavelengths, sinusoidal sound waves and sawtooth shock waves act in almost the same way for particles. This implies that particle acceleration by sawtooth waves works in a similar way as the second-order process for these wavelengths. On the other hand, when the wavelengths of waves get larger, the efficiency of second-order acceleration becomes lower since the velocity difference felt by particles during diffusion becomes smaller. Even for these wavelengths, however, sawtooth waves remain efficient accelerators. We speculate that this efficient acceleration is achieved by crossing weak shock waves many times in the same way as DSA.

\subsection{Particle acceleration by nonlinear sound waves}

Because, by the previous simulations, efficient acceleration by weak shock waves is confirmed at least for some cases, we investigate particle acceleration in the system including both main shock wave and downstream waves. Applying the numerical methods explained in Section 3, the evolution of fluid fields is numerically solved as well as diffusive motion of particles. Fluid equations are solved in the upstream rest frame and shock wave interacts with upstream entropy mode $\rho(x, t)=\rho_{1}+\delta \rho_{1} \sin \left(k_{1} x\right)$ as they propagate. The left panel of Figure 3 is the velocity field at $t=10^{5} \tau_{\mathrm{sc}}\left(p_{0}\right)$ and clearly shows steepening and dissipation of sound waves. In this simulation, shock velocity and amplitude of entropy mode are set to be $u_{\mathrm{sh}}=0.1 \mathrm{c}$ and $\delta \rho_{1}=0.5 \rho_{1}$. The wavelength of entropy mode $k_{1}$ is determined to assure that linear estimate of the wavelength of downstream sound waves becomes $10^{2} \lambda_{\operatorname{mfp}}\left(p_{0}\right)$. 

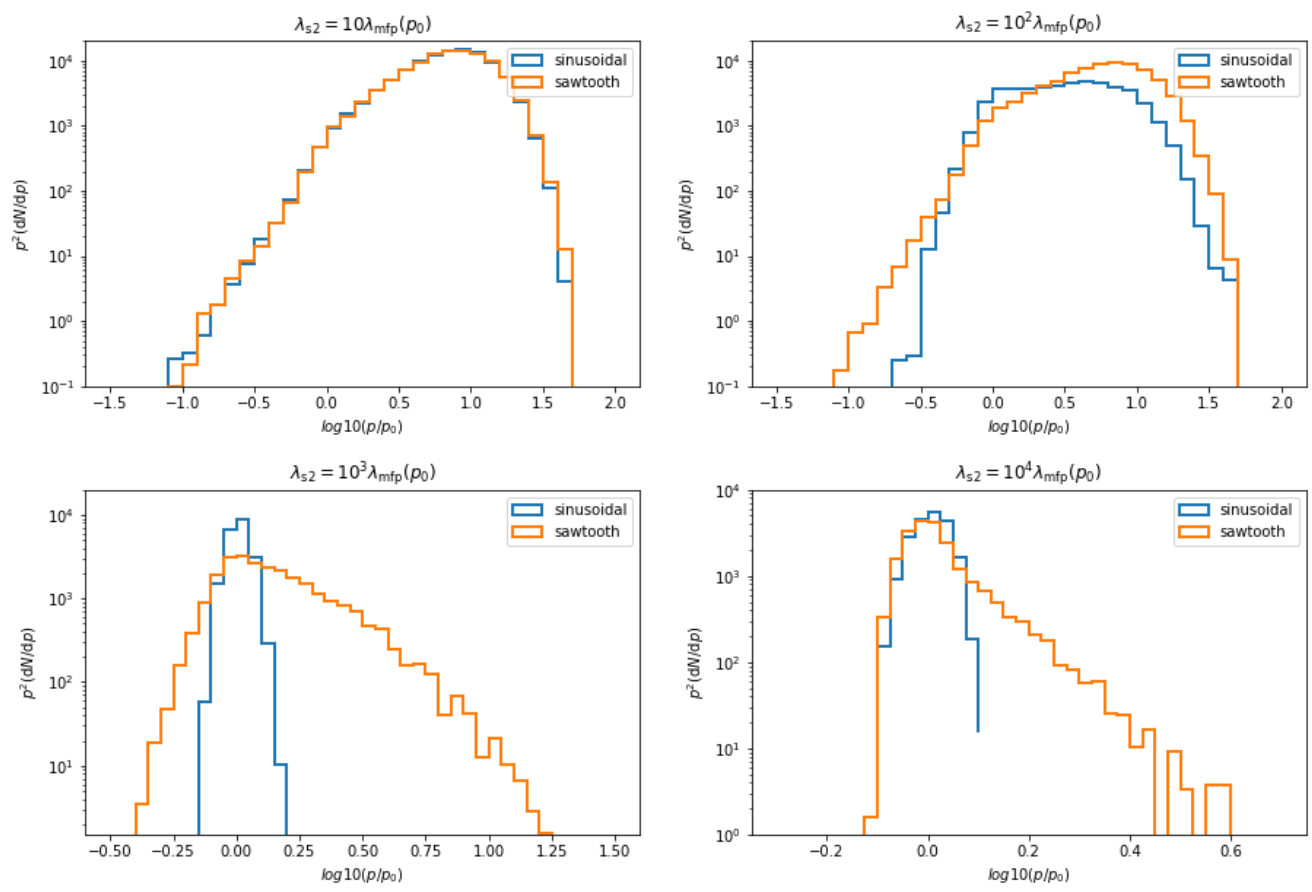

Figure 2: Momentum spectra of particles accelerated by sinusoidal or sawtooth waves at $t=2 \times 10^{5} \tau_{\mathrm{sc}}\left(p_{0}\right)$. The wavelengths are set to be $\lambda_{\delta u_{2}}=10,10^{2}, 10^{3}$, and $10^{4} \lambda_{\mathrm{mfp}}\left(p_{0}\right)$.

The right-hand panel shows the resulted momentum spectrum (orange) and compares that in the case of uniform shock upstream (blue) at $t=10^{5} \tau_{\mathrm{sc}}\left(p_{0}\right)$. Because the number of injected particles is the same for both cases, this indicates that high-energy particles are increased in the case of inhomogeneous upstream. But we cannot see any change in the maximum energy.
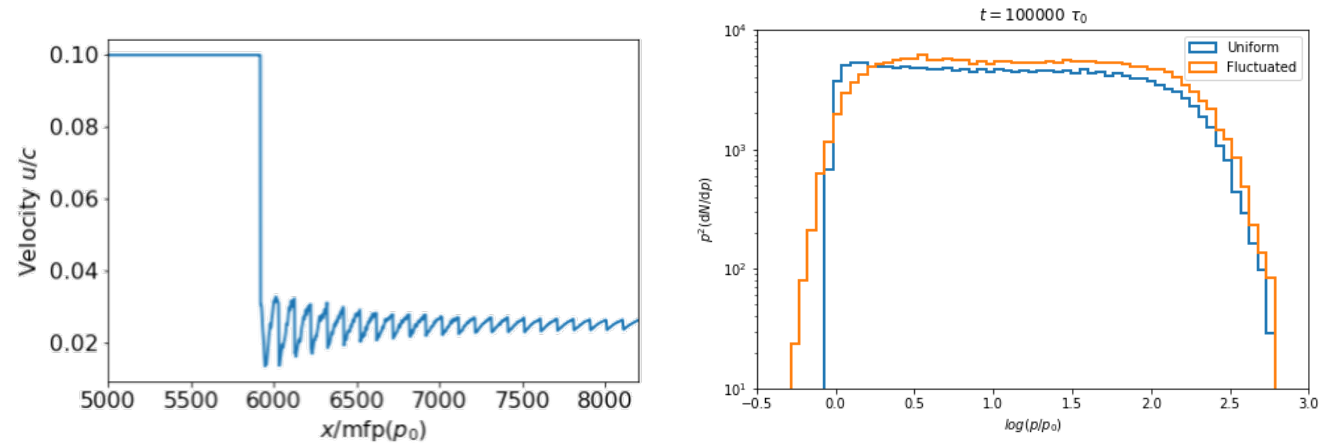

Figure 3: Velocity field (left) and momentum spectrum (right) at $t=10^{5} \tau\left(p_{0}\right)$. 


\section{Discussion}

Figure 2 implies that if there are sound waves whose wavelengths are much larger than particle mean free paths, waves can efficiently accelerate particles by steepening into weak shock waves, while the acceleration is inefficient if they would remain purely sinusoidal waves. Because this acceleration time-scale is shorter than the dissipation time $t_{\text {dis }}$ in the case for $\lambda_{\delta u_{2}}=$ $10^{3} \lambda_{\mathrm{mfp}}\left(p_{0}\right)$, and $10^{4} \lambda_{\mathrm{mfp}}\left(p_{0}\right)$, we expect that spectral modification from the spectrum of DSA certainly occurs for these wavelengths. On the other hand, the spectrum in Figure 3 is for the case where $\lambda_{\delta u_{2}}=10^{2} \lambda_{\mathrm{mfp}}\left(p_{0}\right)$ and dissipation time $t_{\mathrm{dis}}$ is comparable to or less than this. Because even in this case, we find the increase of high-energy particles, we guess that significant modification happens in the case of larger wavelengths or slower shock velocity, where dissipation time becomes longer. However, we met numerical difficulty in doing simulations for those parameters and need to improve our methods. This will be done in our future work(s).

Although our works so far are limited for one-dimensional monochromatic density fluctuations, particle acceleration by turbulence is expected to be much more complex in realistic situations. Considering the oblique incidence of a shock wave, incompressible turbulence is generated and this can also accelerate particles [e.g. 8]. Multi-dimensional fluctuations and cascades of turbulence make situations more complicated. Including the back-reaction of accelerated particles, upstream turbulence or magnetic field might be amplified [e.g. 3] and affect particle acceleration. Because our results show that medium inhomogeneity does modify the energy spectrum of particles even in the simplest case, shock acceleration in an inhomogeneous medium should be investigated further in more realistic situations and compared with observations.

\section{Acknowledgement}

Numerical computations were carried out on the Cray XC50 at the Center for Computational Astrophysics, National Astronomical Observatory of Japan. This work is supported by JSPS KAKENHI grant numbers JP21J20737 (S.Y.), JP19H01893 (Y.O.), and Leading Initiative for Excellent Young Researchers MEXT, Japan.

\section{References}

[1] Armstrong, J. W., Rickett, B. J., \& Spangler, S. R. 1995, Astrophys. J., 443, 209, doi: 10. $1086 / 175515$

[2] Bell, A. R. 1978, Mon. Not. R. Astron. Soc., 182, 147, doi: 10.1093/mnras/182 . 2. 147

[3] Bykov, A. M., Brandenburg, A., Malkov, M. A., \& Osipov, S. M. 2013, Space Sci. Rev., 178, 201, doi: $10.1007 / \mathrm{s} 11214-013-9988-3$

[4] Ferrière, K. 2020, Plasma Phys. Control. Fusion, 62, 1, doi: 10 . 1088/1361-6587/ab49eb

[5] Kirk, J. G., \& Dendy, R. O. 2001, J. Phys. G Nucl. Part. Phys., 27, 1589, doi: 10.1088/ $0954-3899 / 27 / 7 / 316$ 
[6] Malkov, M. A., \& Drury, L. O. 2001, Reports Prog. Phys., 64, 429, doi: 10 . 1088/0034-4885/ $64 / 4 / 201$

[7] Mckenzie, J. F., \& Westphal, K. O. 1968, Phys. Fluids, 11, 2350, doi: 10.1063/1.1691825

[8] Ohira, Y. 2013, Astrophys. J. Lett., 767, 5, doi: 10 . 1088/2041-8205/767/1/L16

[9] Ptuskin, V. S. 1988, Sov. Astron. Lett., 14, 255. https://ui . adsabs.harvard.edu/abs/ 1988SvAL . . . . . 255P/abstract

[10] Stein, R. F., \& Schwartz, R. A. 1972, Astrophys. J., 177, 807, doi: 10 . 1086/151757

[11] Suresh, A., \& Huynh, H. T. 1997, 13th Comput. Fluid Dyn. Conf., 99, 965, doi: 10 .2514/6. 1997-2037

[12] Toro, E. F. 2019, Shock Waves, 29, 1065, doi: 10 . 1007/s00193-019-00912-4

[13] Yokoyama, S., \& Ohira, Y. 2019, 36th ICRC, 358, doi: 10.22323/1.358.0162

[14] Yokoyama, S. L., \& Ohira, Y. 2020, Astrophys. J., 897, 50, doi: 10 . 3847/1538-4357/ab93c3 